\title{
ON FLAT FINITELY GENERATED IDEALS
}

\author{
David E, Dobbs
}

\begin{abstract}
It is shown that if $I$ is a finitely generated ideal of a commutative ring $R$ such that the multiplication map $I \otimes_{R} I \rightarrow I$ is an injection, then $I$ is locally principal. As a corollary, one obtains a new homological characterization of Prüfer domains.
\end{abstract}

The principal application in this note is a homological characterization of Prüfer domains. Recall that a (commutative) integral domain $R$ is said to be a Prüfer domain if and only if $R$ is semihereditary, that is if and only if each nonzero finitely generated ideal of $R$ is invertible; equivalently, if and only if $R_{M}$ is a valuation domain for each maximal ideal $M$ of $R$. One known homological characterization asserts that an integral domain $R$ is a Prüfer domain if and only if each nonzero ideal of $R$ which can be generated by two elements must be invertible ( $c f$. [5, Theorem 22.1]). This characterization is a genuine improvement over the definition, inasmuch as Schülting [11] has recently resolved the "2-generator conjecture" in the negative, by constructing a Prüfer domain with a finitely generated ideal which cannot be generated by two elements. (For earlier work on this conjecture, see [6], [10, Corollary 4.3] and [7, Theorem 3.1].) Now, invertibility of any given nonzero finitely generated ideal $I$ in an integral domain $R$ is equivalent to its being flat (by [4, Theorem 1], see also [1, Theorem 1 , p. 109]); and hence is equivalent to requiring that the multiplication map $I \otimes_{R} J \rightarrow I$ is an injection for each finitely generated ideal $J$ of $R$.

Received 16 August 1979. 
(This characterization of $R$-flatness of $I$ is due essentially to Serre and may be extracted from [1, Proposition 1, p. 37]. Another approach to it follows by combining the title result of [8] with Baer's criterion for injective modules.) Our main result on flatness, which is stated next, asserts that the only " $J$ " which need be tested is $I$ itself.

PROPOSITION 1. Let $I$ be a finitely generated ideal of an integral domain $R$. Then $I$ is $R$-flat if and only if the multiplication map $I \otimes_{R} I \rightarrow I$ is an injection.

The condition in Proposition 1 may be recast in other terms. The requirement that an ideal I of an integral domain $R$ incur a monomorphic multiplication $I \otimes_{R} I+I$ is, of course, equivalent to requiring that $\operatorname{Tor}_{I}^{R}(I, R / I)=0$; by a proof of Chase (see (c) $\Rightarrow$ (d) in [2, Theorem 4.2]), this condition is also equivalent to requiring that $I \otimes_{R} I$ be a torsion-free $R$-module. We shall postpone the proof of Proposition $I$, in order to next present the promised application.

COROLLARY. An integraz domain $R$ is a Prüfer domain if and only if $I \otimes_{R} I$ is a torsion-free R-module for each ideal $I$ of $R$ which can be generated by two elements.

Proof of Corollary. By the above comments, $R$ is a Prüfer domain if and only if $I$ is $R$-flat for each ideal $I$ of $R$ which can be generated by two elements. Thus, an application of (the recast) Proposition I completes the proof.

The effect of the preceding result is to reduce the set of multiplication maps $I \otimes_{R} J \rightarrow I$ whose kernels need to be checked in order to verify that a given integral domain $R$ is a Prüfer domain. It seems worthwhile to observe an analogous reduction involving overrings. By (the proof of) a result of Richman [9, Lemma 4], an integral domain $R$ is a Prüfer domain if and only if $R[u]$ is $R$-flat for each nonzero element $u$ in the quotient field $K$ of $R$. Although testing $R$-flatness for all such $R[u]$ entails showing that $R[u] \otimes_{R} E \rightarrow R[u] \otimes_{R} F$ is an injection for all (such $u$ and) $R$-modules $E \subset F$, a proof by Davis [3, Theorem] reduces the task to establishing that each multiplication map 
$R[u] \otimes_{R} R\left[u^{-1}\right] \rightarrow K \quad$ is an injection.

The earlier comments serve to dispatch the "only if" half of Proposition 1. To prove the "if" half, it evidently suffices to establish the following more general result, since flatness is a local property.

PROPOSITION 2. If $I$ is a finitely generated ideal of a commitative ring $R$ such that the multiplication map $I \otimes_{R} I \rightarrow I$ is an injection, then $I$ is locally principal.

Proof. Standard localization techniques (cf. [1]) permit us to suppose that $R$ is quasilocal, say with (unique) maximal ideal $M$. By hypothesis, $I=R a_{1}+\ldots+R a_{n}$, for suitable elements $a_{i}$ such that $n$ is minimal. We shall suppose $n>1$ and seek a contradiction.

Consider the short exact sequence of $R$-modules

$$
0 \rightarrow L \rightarrow R \oplus \ldots \oplus R \stackrel{f}{\rightarrow} I \rightarrow 0 \text {, }
$$

where $f$ is given by

$$
f\left(r_{1}, \ldots, r_{n}\right)=\sum_{k} r_{k} a_{k}
$$

and $L=\operatorname{ker}(f)$. Applying the functor $I \otimes_{R}$. leads to the first row of the following commutative diagram, whose rows are exact:

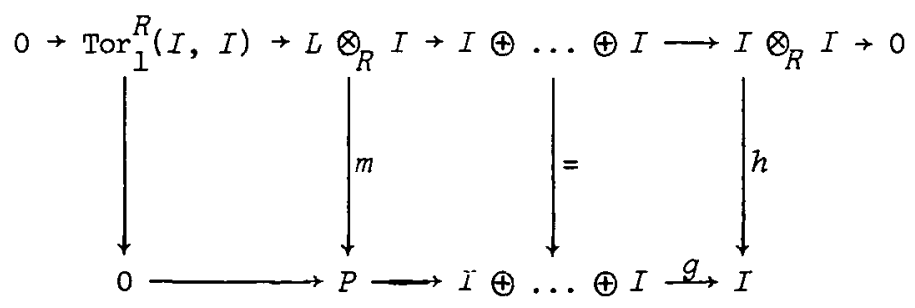

(Specifically, $h$ is the multiplication map; $g$ is the restriction of $f ; P=\operatorname{ker}(g)$; and $m$ is induced by diagram chasing.) An application of the "snake lemma" [1, Proposition 2 (iii), p. 6] produces an exact sequence

$$
0 \rightarrow \operatorname{ker}(h) \rightarrow \operatorname{coker}(m) \rightarrow 0 ;
$$

that is, $\operatorname{ker}(h) \cong \operatorname{coker}(m)$. However, by hypothesis, $h$ is an injection, 
so that $\operatorname{ker}(h)=0$ and $m$ is surjective. In particular, the element

$$
p=\left(a_{n}, a_{n}, \ldots, a_{n},-\left(a_{1}+\ldots+a_{n-1}\right)\right)
$$

is in the image of $m$ (since commutativity of $R$ guarantees that $p \in P$ ). Thus,

$$
p=m\left(\sum_{j}\left(r_{1 j}, \ldots, r_{n j}\right) \otimes i_{j}\right)
$$

for finitely many suitable elements $\left(r_{1 j}, \ldots, r_{n j}\right) \in L$ and $i_{j} \in I$. Notice first that $r_{n j} \in M$ for each $j$, for otherwise one would infer from $r_{1 j} a_{1}+\ldots+r_{n j} a_{n}=0$ that

$$
a_{n}=-r_{n j}^{-1}\left(r_{1 j} a_{1}+\ldots+r_{n-1, j} a_{n-1}\right) \text {, }
$$

contradicting the minimality of $n$. Moreover, by the construction of $m$, we have

$$
-\left(a_{1}+\ldots+a_{n-1}\right)=\sum_{j} r_{n j} i_{j}
$$

Accordingly, if each $i_{j}$ is expressed as a linear combination of $a_{1}, \ldots, a_{n}$, rearranging the preceding equation leads to an element $d \in M$ such that $(1-d) a_{1} \in R a_{2}+\ldots+R a_{n}$. Since $I-d$ is a unit of $R$, this contradicts the minimality of $n$, and completes the proof.

Proposition 2 sharpens a recent result of Smith [12, Corollary of Theorem 2], by eliminating Smith's hypothesis that 2 is a unit of $R$. Note that Proposition 1 is essentially equivalent to the case $s=t=1$ of [12, Lemma 2], although their proofs are rather different.

In closing, we note that the proof of Proposition 2 does not work if $R$ is noncommutative (and $I$ is a two-sided ideal). It would be of interest to find analogues for this case.

Note added in Proof (26 November 1979). Proposition 2 may also be proved by reasoning as in [12, Lemma 2]. W. Vasconcelos has kindly communicated to the author another proof of Proposition 2 based on the following observation. If the minimal number of generators of a finitely generated ideal $I$ of a quasi-local commutative ring $R$ is 
$v(I)=n \geq 1$, then $v\left(I \otimes_{R} I\right)=n^{2}$ and $v\left(I^{2}\right) \leq\left(\begin{array}{c}n+1 \\ 2\end{array}\right)$. One may prove similarly that a finitely generated ideal $I$ of a commutative ring $R$ is locally principal if the multpilication map $I \otimes_{R}(R a+R b) \rightarrow I$ is an injection for each $a$ and $b$ in $I$.

\section{References}

[1] Nicolas Bourbaki, Elements of mathematics. Commative algebra (Hermann, Paris; Addison-Wesley, Reading, Massachusetts, 1972).

[2] Stephen U. Chase, "Direct products of modules", Trans. Amer. Math. Soc. 97 (1960), 457-473.

[3] Edward D. Davis, "A remark on Prüfer rings", Proc. Amer. Math. Soc. 20 (1969), 235-237.

[4] Shizuo Endo, "On flat modules over commutative rings", J. Math. Soc. Japan 14 (1962), 284-291.

[5] Robert W. Gilmer, Multiplicative ideal theory, Revised edition (Pure and Applied Mathematics, 12. Marcel Dekker, New York, 1972).

[6] Robert Gilmer and William Heinzer, "On the number of generators of an invertible ideal", J. Algebra 14 (1970), 139-151.

[7] Raymond C. Heitmann, "Generating ideals in Prüfer domains", Pacific J. Math. 62 (1976), 117-126.

[8] J. Lambek, "A module is flat if and only if its character module is injective", Canad. Math. Buzl. 7 (1964), 237-243.

[9] Fred Richman, "Generalized quotient rings", Proc. Amer. Math. Soc. 16 (1965), 794-799.

[10] Judith D. Sally and Wolmer V. Vasconcelos, "Stable rings", J. Pure App Z. Algebra 4 (1974), 319-336.

[11] Heinz-Werner Schülting, "Über die Erzeugendenanzahl invertierbarer Ideale in Prüferringen", Comn. Algebra 7 (1979), 1331-1349.

[12] William W. Smith, "Invertible ideals and overrings", Houston J. Math. 5 (1979), 141-153.

Department of Mathematics, University of Tennessee, Knoxville, Tennessee 37916, USA. 